Draft version February 1, 2018

Preprint typeset using $\mathrm{ATTEX}_{\mathrm{E}}$ style emulateapj v. 01/23/15

\title{
ELUCIDATING $\Lambda$ CDM: IMPACT OF BARYON ACOUSTIC OSCILLATION MEASUREMENTS ON THE HUBBLE CONSTANT DISCREPANCY
}

\author{
G. E. Addison ${ }^{1}$, D. J. Watts ${ }^{1}$, C. L. Bennetr ${ }^{1}$, M. Halpern ${ }^{2}$, G. Hinshaw ${ }^{2}$, And J. L. Weiland ${ }^{1}$ \\ Draft version February 1, 2018
}

\section{ABSTRACT}

\begin{abstract}
We examine the impact of baryon acoustic oscillation (BAO) scale measurements on the discrepancy between the value of the Hubble constant $\left(H_{0}\right)$ inferred from the local distance ladder and from Planck cosmic microwave background (CMB) data. While the BAO data alone cannot constrain $H_{0}$, we show that combining the latest BAO results with WMAP, Atacama Cosmology Telescope (ACT), or South Pole Telescope (SPT) CMB data produces values of $H_{0}$ that are $2.4-3.1 \sigma$ lower than the distance ladder, independent of Planck, and that this downward pull was less apparent in some earlier analyses that used only angle-averaged BAO scale constraints rather than full anisotropic information. At the same time, the combination of BAO and CMB data also disfavors the lower values of $H_{0}$ preferred by the Planck high-multipole temperature power spectrum. Combining galaxy and Lyman- $\alpha$ forest $(\mathrm{Ly} \alpha) \mathrm{BAO}$ with a precise estimate of the primordial deuterium abundance produces $H_{0}=66.98 \pm 1.18 \mathrm{~km} \mathrm{~s}^{-1} \mathrm{Mpc}^{-1}$ for the flat $\Lambda \mathrm{CDM}$ model. This value is completely independent of CMB anisotropy constraints and is $3.0 \sigma$ lower than the latest distance ladder constraint, although $2.4 \sigma$ tension also exists between the galaxy $\mathrm{BAO}$ and $\mathrm{Ly} \alpha \mathrm{BAO}$. These results show that it is not possible to explain the $H_{0}$ disagreement solely with a systematic error specific to the Planck data. The fact that tensions remain even after the removal of any single data set makes this intriguing puzzle all the more challenging to resolve.
\end{abstract}

Keywords: cosmic background radiation - cosmological parameters - cosmology: observations distance scale - large-scale structure of universe

\section{INTRODUCTION}

While no single data set currently provides compelling evidence for a deviation from the standard Lambda cold dark matter $(\Lambda \mathrm{CDM})$ cosmological model, the values of some parameters inferred from different measurements now exhibit moderate to severe tension. This is most pronounced in the value of the Hubble constant, $H_{0}$. Riess et al. (2016; hereafter R16) provided the most recent and most precise local distance ladder constraint, finding $H_{0}=73.24 \pm 1.74 \mathrm{~km} \mathrm{~s}^{-1} \mathrm{Mpc}^{-1}$ by combining three absolute distance anchors with the empirical period-luminosity relation for Cepheid variable stars and the relationship between observed light curve and intrinsic luminosity of type Ia supernovae (SNe). The most precise $H_{0}$ prediction from cosmic microwave background (CMB) anisotropy power spectrum measurements is currently provided by the Planck mission. The 2015 Planck temperature and polarization analysis produced $H_{0}=$ $67.31 \pm 0.96 \mathrm{~km} \mathrm{~s}^{-1} \mathrm{Mpc}^{-1}$ (Planck Collaboration et al. 2016). An updated analysis with a revised estimate of the optical depth to reionization, $\tau$, found $H_{0}=66.88 \pm 0.91$, or $66.93 \pm 0.62$ if preliminary small-scale polarization data are also included (Planck Collaboration Int. XLVI 2016). Assuming all uncertainties are Gaussian, these values are, respectively, 3.0, 3.2, and $3.4 \sigma$ lower than the distance ladder determination. Strong lensing timing delay measurements have produced $H_{0}$ constraints consis- tent with the distance ladder, and in mild tension with Planck (Bonvin et al. 2017). Tensions also exist between the Planck predictions for the growth of cosmic structure (through the matter density, $\Omega_{m}$, and present-day density fluctuation amplitude, $\sigma_{8}$ ) and measurements using weak gravitational lensing (e.g., Hildebrandt et al. 2017 Joudaki et al. 2017; Alsing et al. 2017; Köhlinger et al. 2017).

It is not clear whether the problem is with the model or the data. While it is certainly plausible that a failure of the standard model could show up as a discrepancy between the CMB and low-redshift measurements, none of the commonly-considered or physically-motivated extensions to $\Lambda \mathrm{CDM}$ appear to provide a convincing improvement when considering the full range of data available (e.g., Planck Collaboration et al. 2016, Bernal et al. 2016). In principle, the CMB prediction for $H_{0}$ could be significantly increased by modifying the expansion history of the universe post-recombination, for example by allowing spatial curvature or a dark energy equation of state $w \neq-1$. Planck temperature and polarization data alone mildly prefer a non-zero curvature, but $H_{0}$ goes in the wrong direction. The Planck $2015 \Lambda \mathrm{CDM}+\Omega_{k}$ constraint is $53.2 \pm 5.1 \mathrm{~km} \mathrm{~s}^{-1} \mathrm{Mpc}^{-1}$ (mean and standard deviation), with $95 \%$ of Markov chain Monte Carlo (MCMC) samples lying in $43.7<H_{0} / \mathrm{km} \mathrm{s}^{-1} \mathrm{Mpc}^{-1}<$ 63.5. Allowing $w<-1$ can shift the Planck prediction to 70 or even $80 \mathrm{~km} \mathrm{~s}^{-1} \mathrm{Mpc}^{-1}$, however, even leaving aside questions of the physical interpretation of $w<-1$, resolving the $H_{0}$ disagreement with evolution in $w$ is strongly disfavored when we include ob-

\footnotetext{
3 The public Planck MCMC chains can be downloaded from the Planck legacy archive: http://pla.esac.esa.int/pla/
} 
servations of the expansion rate, such as baryon acoustic oscillations (BAO) in the clustering of galaxies, or high-redshift SNe. Alam et al. (2017) combined Planck data with the latest galaxy clustering and SNe data and found $H_{0}=67.9 \pm 0.9 \mathrm{~km} \mathrm{~s}^{-1} \mathrm{Mpc}^{-1}$ for constant $w$, or $67.5 \pm 1.0 \mathrm{~km} \mathrm{~s}^{-1} \mathrm{Mpc}^{-1}$ for the $w_{0}-w_{a}$ parameterization.

Modifying the early-universe expansion history, for instance by increasing the number of effective neutrino species, $N_{\text {eff }}$, can increase the CMB $H_{0}$ prediction. The Planck data do not favor this solution, for example Alam et al. (2017) report $N_{\text {eff }}=2.97 \pm 0.20$ (Planck-only), and $3.03 \pm 0.18$ (Planck plus galaxy clustering), consistent with the standard model value of 3.046, with corresponding $H_{0}$ constraints of $66.6 \pm 1.6$ and $67.5 \pm$ $1.2 \mathrm{~km} \mathrm{~s}^{-1} \mathrm{Mpc}^{-1}$. Adding $N_{\text {eff }}$ in these fits shifted the tension with the distance ladder from $3.2 \sigma$ to $2.8 \sigma$ (Planck-only) and from $3.1 \sigma$ to $2.7 \sigma$ (Planck plus galaxy clustering). A fit to the 2015 Planck temperature and polarization data plus BAO fixing $N_{\text {eff }}=3.4$, the value found by R16 to most effectively relieve Planck-distance ladder tension, leads to an increase in the parameter combination best constrained by weak lensing measurements, $\sigma_{8} \Omega_{m}^{0.5}$, by around $1.5 \%, 0.8$ times the original uncertainty 4 This slightly worsens the tension between Planck and the weak lensing analyses mentioned above, which found $\sigma_{8} \Omega_{m}^{0.5}$ values lower than Planck at the $2-3 \sigma$ level when the standard model was assumed. Brust et al. (2017) found that the Planck-lensing consistency could be improved by also introducing some degree of neutrino or dark radiation self-interaction, but, even with a second additional parameter, a joint fit to the Planck, BAO, distance ladder, weak lensing, and galaxy cluster data produced a $H_{0}$ distribution peaking at $69.95 \mathrm{~km} \mathrm{~s}^{-1} \mathrm{Mpc}^{-1}$, still almost $2 \sigma$ lower than the R16 measurement. In short, while a non-standard value of $N_{\text {eff }}$ cannot be ruled out, its inclusion is not justified by the improvements to the fit.

On the other hand, the discrepant data sets have passed a range of systematic checks. The R16 distance ladder analysis used infrared data to greatly reduce the effects of reddening, substituted rungs of the ladder with alternative data, compared different calibrators, corrected for estimated local motion, and constructed a systematic error budget from considering a range of modeling variants (see also, e.g., Cardona et al. 2017; Zhang et al. 2017; Feeney et al. 2017; Dhawan et al. 2017, Follin \& Knox 2017). The distance ladder measurements have substantially improved since the analysis by Efstathiou (2014). While the constraints have become tighter, the mean $H_{0}$ values in recent years have remained fairly constant (e.g., Riess et al. 2009, 2011, Freedman et al. 2012). Likewise, the Planck team has performed an array of robustness checks of their data, investigating the effects of detector nonlinearity, beam shapes and sidelobes, and various other calibration-related issues. Also, the preference for a lower $H_{0}$ from Planck does not appear to be driven by a particular frequency channel (Planck Collaboration Int. LI 2016).

Ultimately it may take additional high-precision mea-

\footnotetext{
4 This result is taken from the Planck 2015 base $\mathrm{Pl} i \mathrm{kHM}$ TTEE lowTEB post $\mathrm{BAO}$ base_nnu_plikHM_TTTEEE_lowTEB_nnup39_BAO chains.
}

surements to shed light on what is really going on. More precise measurements may bring with them new tensions or disagreements, and the handling of systematic errors will get harder, not easier, as statistical uncertainties are reduced. In the meantime, it is therefore helpful to reexamine existing data and ask whether any extra insight into the discrepancies can be gleaned. To this end, in this paper we investigate in detail the indirect but important role played by $\mathrm{BAO}$ measurements in $H_{0}$ constraints, both with and without $\mathrm{CMB}$ anisotropy data. While this topic has been addressed in the literature, we describe several results that have either not previously been discussed, or are not widely appreciated. In Section 2, we review the BAO measurements. In Section 3, we describe results of fitting cosmological parameters to $\mathrm{BAO}$ in conjunction with other data sets, focussing on $H_{0}$. A discussion and conclusions follow in Sections 4 and 5 .

\section{BAO MEASUREMENTS}

The first convincing detections of the BAO feature in the correlation function or power spectrum of large-scale structure (LSS) tracers were made a little over a decade ago (Eisenstein et al. 2005, Cole et al. 2005). Since that time, deeper surveys with orders of magnitude more galaxies, notably the Baryon Oscillation Spectroscopic Survey (BOSS ${ }^{5}$, have led to both improved precision in the BAO scale measurements over a range of redshifts, and improved analysis methodologies (e.g., Percival et al. 2014; Anderson et al. 2014; Kazin et al. 2014 Reid et al. 2016). While current and future BAO surveys are proposed as a means of improving dark energy constraints, $\mathrm{BAO}$ measurements also provide significant information about parameters in the standard $\Lambda$ CDM model, particularly in joint fits with the CMB.

A detailed discussion of BAO physics can be found in Chapter 4 of Weinberg et al. (2013). The BAO scale in the transverse and line-of-sight direction correspond to measurements of $D_{M}(z) / r_{d}$ and $H(z) r_{d}$, where $D_{M}(z)=$ $(1+z) D_{A}(z)$ is the comoving angular diameter distance at the effective redshift of the survey and $r_{d}$ is the sound horizon at the drag epoch where baryons decouple from photons, denoted $z_{d}$. The sound horizon is defined as

$$
r_{d}=\int_{z_{d}}^{\infty} d z \frac{c_{s}(z)}{H(z)},
$$

where the sound speed, $c_{s}=c / \sqrt{3(1+R)}$, depends on the ratio of baryon to photon density, with $R=3 \rho_{b} / 4 \rho_{\gamma}$. The sound horizon is sensitive to the physics of the early universe, including the pre-recombination expansion history and the number of effective neutrino species, $N_{\text {eff }}$, while $D_{M}(z)$ and $H(z)$ at the effective redshift of the survey depend on the late-time expansion.

In some cases, only a joint constraint, for example on $D_{V}(z) / r_{d}$, where $D_{V}(z)=\left[c z D_{M}^{2}(z) / H(z)\right]^{1 / 3}$, is provided, representing an angle-averaged constraint. This can be helpful where the BAO feature is detected at lower significance and the separate line-of-sight and transverse

5 http://www.sdss3.org/surveys/boss.php

6 The sound horizon was referred to as $r_{s}$ by Addison et al. (2013), we have adopted the $r_{d}$ notation here for consistency with other work. 
Table 1

BAO measurements used in this work

\begin{tabular}{|c|c|c|c|c|c|}
\hline Dataset & LSS tracer & $z_{\mathrm{eff}}$ & Measurement ${ }^{a}$ & 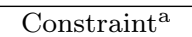 & Reference \\
\hline 6dFGS & galaxies & 0.106 & $r_{d} / D_{V}\left(z_{\mathrm{eff}}\right)$ & $0.336 \pm 0.015$ & Beutler et al. $(2011)$ \\
\hline SDSS MGS & galaxies & 0.15 & $D_{V}\left(z_{\text {eff }}\right) r_{d, \text { fid. }} / r_{d}[\mathrm{Mpc}]$ & $664 \pm 25$ & Ross et al. 2015 \\
\hline BOSS DR12 & galaxies & $\begin{array}{l}0.38 \\
0.51\end{array}$ & $\begin{array}{l}D_{M}\left(z_{\text {eff }}\right) r_{d, \text { fid. }} / r_{d}[\mathrm{Mpc}] \\
H\left(z_{\text {eff }}\right) r_{d} / r_{d, \text { fid. }}\left[\mathrm{km} \mathrm{s}^{-1} \mathrm{Mpc}^{-1}\right] \\
D_{M}\left(z_{\text {eff }}\right) r_{d, \text { fid. }} / r_{d}[\mathrm{Mpc}] \\
H\left(z_{\text {eff }}\right) r_{d} / r_{d, \text { fid. }}\left[\mathrm{km} \mathrm{s}^{-1} \mathrm{Mpc}^{-1}\right] \\
D_{M}\left(z_{\text {eff }}\right) r_{d, \text { fid. }} / r_{d}[\mathrm{Mpc}] \\
H\left(z_{\text {eff }}\right) r_{d} / r_{d, \text { fid. }}\left[\mathrm{km} \mathrm{s}^{-1} \mathrm{Mpc}^{-1}\right]\end{array}$ & $\begin{array}{l}1512 \pm 25 \\
81.2 \pm 2.4 \\
1975 \pm 30 \\
90.9 \pm 2.3 \\
2307 \pm 37 \\
99.0 \pm 2.5\end{array}$ & Alam et al. (2017) \\
\hline BOSS DR11 Ly $\alpha$ & Ly $\alpha$ absorber ${ }^{\natural}$ & 2.34 & $\begin{array}{l}D_{A}\left(z_{\mathrm{eff}}\right) / r_{d} \\
c /\left[H\left(z_{\mathrm{eff}}\right) r_{d}\right]\end{array}$ & $\begin{array}{c}11.28 \pm 0.65 \\
9.18 \pm 0.28\end{array}$ & Delubac et al. (2015) \\
\hline BOSS DR11 QSO $\times$ Ly $\alpha$ & QSO, Lya & 2.36 & $\begin{array}{l}D_{A}\left(z_{\text {eff }}\right) / r_{d} \\
c /\left[H\left(z_{\text {eff }}\right) r_{d}\right]\end{array}$ & $\begin{array}{c}10.8 \pm 0.4 \\
9.0 \pm 0.3\end{array}$ & Font-Ribera et al. (2014) \\
\hline
\end{tabular}

${ }^{a}$ Note that the fiducial sound horizon, $r_{d \text {,fid. }}$, differs across different analyses. We provide constraints here only to show relative precision. For parameter fitting we use full likelihood surfaces, including correlations across the BOSS redshift bins or between $D_{M}$ and $H$.

${ }^{\mathrm{b}}$ For brevity we refer to the $\operatorname{Ly} \alpha$ and $\mathrm{QSO} \times \operatorname{Ly} \alpha$ measurements collectively as Ly $\alpha$.

measures are poorly constrained or have distributions with non-Gaussian tails. Whenever possible, we use the joint anisotropic $D_{M}(z) / r_{d}$ and $H(z) r_{d}$ constraints. Quantities like $D_{V}(z) / r_{d}$ entail a compression of information that potentially give a false sense of agreement with other data, as discussed in Section 3.2.

\subsection{Current BAO constraints}

The BAO data sets included in fits presented in this paper are listed in Table 1. For the 6dF Galaxy Survey (6dFGS) and Sloan Digital Sky Survey (SDSS) Main Galaxy Sample (MGS), we adopt a simple Gaussian likelihood for $r_{d} / D_{V}(z)$ or $D_{V}(z) / r_{d}$. Away from the peak of the likelihood these constraints become non-Gaussian, however the uncertainties for these measurements are large enough that the preferred model solutions never lie far from the peak in a joint fit with other data. We use the consensus BAO scale measurements from the BOSS Data Release 12 (DR12), including $D_{M}(z) / r_{d}$ and $H(z) r_{d}$ for each of the three redshift bins and the six-bysix covariance matrix described by Alam et al. (2017). We restrict our analysis to the $\mathrm{BAO}$ scale as it is the most robust observable from LSS surveys (e.g., Weinberg et al. 2013, and references therein), and do not consider redshift-space distortion constraints or information from the broadband correlation function. We do not include results from the Wiggle $Z^{7}$ survey, which are consistent with BOSS and partially overlap in sky coverage (Beutler et al. 2016).

$\mathrm{BAO}$ have been measured in the Lyman- $\alpha$ (Ly $\alpha$ ) forest of BOSS quasars (QSOs), and in the cross-correlation between the QSOs and Ly $\alpha$ absorbers, at effective redshifts of $2.3-2.4$ (Busca et al. 2013; Slosar et al. 2013, Font-Ribera et al. 2014; Delubac et al. 2015: Bautista et al. 2017). BAO measurements at these redshifts, when the dark energy contribution to the total energy budget of the universe is small, are a powerful complement to the BAO from lower-redshift galaxy surveys. The analysis methodology and systematic error treatment required

${ }^{7}$ http://wigglez.swin.edu.au/site/ to extract the Ly $\alpha$ BAO scale are less mature than for the galaxy BAO and are an active field of research (e.g., Blomqvist et al. 2015). The anisotropic BAO measurements from the DR11 Ly $\alpha$ and $\mathrm{QSO} \times \mathrm{Ly} \alpha$ analyses are in $\sim 2.5 \sigma$ tension with Planck predictions assuming a standard flat $\Lambda$ CDM model. This tension was reduced slightly in the DR12 Ly $\alpha$ BAO analysis (Bautista et al. 2017). Bautista et al. (2017) found that the shift in the DR12 Ly $\alpha$ constraints was predominantly due to the additional data rather than some different treatment of systematic effect: 8 . We present results using the DR11 Ly $\alpha$ and $\mathrm{QSO} \times \mathrm{Ly} \alpha$ constraints, and from combining the galaxy and $\mathrm{Ly} \alpha \mathrm{BAO}$, noting that $\sim 2.5 \sigma$ effects can and do arise purely from statistical fluctuations, and that there is currently no known systematic error that explains this tension.

Other BAO measurements have been reported, for example using galaxy clusters as LSS tracers (e.g., Veropalumbo et al. 2016, Hong et al. 2016). These results are generally less precise than the galaxy BAO, at similar redshifts, and their inclusion would not significantly affect our results. Recently, the first measurement of BAO from the extended Baryon Oscillation Spectroscopic Survey (eBOSS ${ }^{9}$ was reported using clustering of quasars at $0.8 \leq z \leq 2.2$ (Ata et al. 2017). BAO constraints from this redshift range are potentially a useful addition to the galaxy and $\mathrm{Ly} \alpha \mathrm{BAO}$ and upcoming, higher-precision eBOSS measurements will be interesting to include in future analyses.

\subsection{Choice of $C M B$ data for joint fits}

Joint fits between Planck and BAO have been reported extensively for a range of cosmological models in recent work (e.g., Aubourg et al. 2015, Planck Collaboration et al. 2016, Alam et al. 2017). While Planck provides the most precise $\mathrm{CMB}$ constraints, $\sim 2.5 \sigma$ tension exists

8 The DR12 QSO $\times$ Ly $\alpha$ analysis, released while this work was in review, produced results consistent with DR11, in tension with Planck predictions at the $2.3 \sigma$ level du Mas des Bourboux et al. 2017)

9 http://www.sdss.org/surveys/eboss/ 
between determination of some parameters from splitting the Planck power spectrum into multipoles $\ell<800$ and $\ell>800$, where the choice of 800 corresponds to a roughly even division of overall constraining power $\mathrm{Ad}-$ dison et al. 2016). In the full $\Lambda$ CDM model space, the tension is not significant $(1.8 \sigma$ for the assumptions used by Addison et al. 2016; see also Planck Collaboration Int. LI 2016). Current low-redshift cosmological observations do not provide strong constraints across the full $\Lambda$ CDM parameter space, however they do provide independent and precise constraints on a subset of parameters, including $H_{0}, \Omega_{m}$, and $\sigma_{8}$. These parameters are therefore of particular interest when it comes to assessing the performance of the $\Lambda \mathrm{CDM}$ model and testing for alternatives. Given the moderate internal Planck tension in these parameters, it is informative to consider other CMB measurements to help understand the extent to which conclusions are driven by Planck data, or are independent of Planck. In this work we therefore also include results from the final WMAP 9-year analysis (Bennett et al. 2013; Hinshaw et al. 2013), the Atacama Cosmology Telescope polarization-sensitive receiver (ACTPol; Thornton et al. 2016; Louis et al. 2017; Sherwin et al. 2016) two-season survey, covering $548 \mathrm{deg}^{2}$, and the $2500 \mathrm{deg}^{2}$ South Pole Telescope Sunyaev-Zel'dovich survey (SPT-SZ; Carlstrom et al. 2011; Story et al. 2013; van Engelen et al. 2012).

\section{RESULTS}

\subsection{Combining $B A O$ and $C M B$ anisotropy measurements}

In Table 2 we show $H_{0}$ constraints within the $\Lambda$ CDM model from $\mathrm{CMB}$ data sets with and without the inclusion of the BAO data. ACTPol and SPT use WMAP or Planck data only to provide an absolute calibration, that is, a single scale-independent multiplicative rescaling of the measured power spectrum. Since these experiments do not measure $\tau$, we adopt a Gaussian prior, either the same broader $\tau=0.07 \pm 0.02$ prior used by Planck Collaboration XI (2016) and Addison et al. $(2016)$, or the $\tau=0.055 \pm 0.009$ constraint from the latest Planck HFI low- $\ell$ polarization determination (Planck Collaboration Int. XLVI 2016). Here and throughout this paper we quote the mean and standard deviation from MCMC runs using the CosmoMd ${ }^{10}$ package (Lewis \& Bridle 2002), with convergence criterion $R-1<0.01$ (Gelman \& Rubin 1992). Since we are not investigating foreground modeling in this work we use foregroundmarginalized CMB likelihood codes for ACTPol and SPT (Dunkley et al. 2013; Calabrese et al. 2013). Uncertainties in foreground and other nuisance parameters propagate to cosmological parameters through an increase in power spectrum uncertainties in these codes. In the Planck rows of Table 2 we include the exact name of the likelihood file for clarity since a range of likelihoods have been provided by the Planck collaboration. These likelihoods include Planck foreground and nuisance parameters as described by Planck Collaboration XI (2016). We show results with and without CMB lensing power spectra (denoted ' $\phi \phi$ ' in the third column of Table 2 ), noting that the lensing measurements have a moderate effect on

10 http://cosmologist.info/cosmomc/ some of the CMB-only constraints but reduced impact when the BAO are included. In the last four rows of Table 2 we also list constraints from splitting the Planck temperature power spectrum at $\ell=800$ (Addison et al. 2016), as mentioned in Section 2.2 and discussed further in Section 4.

Adding galaxy BAO to any of the CMB measurements listed in Table 2 substantially tightens the $H_{0}$ prediction, by more than a factor of three in the case of ACTPol or SPT. While there is still scatter in the CMB + galaxy BAO $H_{0}$ values, the spread is substantially reduced compared to the CMB-only column. The ACTPol $+\mathrm{BAO}$ and $\mathrm{SPT}+\mathrm{BAO}$ combinations produce $H_{0}$ constraints of comparable precision to Planck alone. The synergy between the galaxy $\mathrm{BAO}$ and $\mathrm{CMB}$ measurements for $\Lambda \mathrm{CDM}$ is illustrated in Figure 1 using the BOSS DR12 anisotropic BAO measurements at $z_{\text {eff }}=0.61$ as an example. The predictions from the CMB are shown with MCMC samples color-coded by $H_{0}$, which varies fairly monotonically along the degeneracy line set by the angular acoustic scale, corresponding to the peak spacing in the $\mathrm{CMB}$ power spectrum. The MCMC samples shown are drawn from the full chains, and include points from the tails of the distributions in addition to high-likelihood samples. The shaded blue contours correspond to the BOSS measurements, which are precise enough to substantially reduce the range of $H_{0}$ values allowed by breaking CMB degeneracies. The mixing of colors visible in the ACTPol and SPT panels reflects additional degeneracy between $H_{0}$ and other parameters arising from the more limited range of angular scales provided by these data.

In conjunction with the CMB, and in the context of $\Lambda \mathrm{CDM}$, the BAO have the effect of disfavoring both the higher values of $H_{0}$ preferred by the distance ladder, and the lower values preferred by the Planck damping tail at $\ell>800$. If we exclude Planck, the $\mathrm{CMB}+\mathrm{BAO}$ values lie $2.4-3.1 \sigma$ from the R16 measurement, depending on the choice of CMB dataset. While this trend has been reported before using WMAP data (Planck Collaboration et al. 2016, Bernal et al. 2016), here we show that the measurements of the damping tail from ACTPol and SPT produce the same effect even without information from the larger scales measured by the satellite experiments. The fact that combining ACTPol and BAO data produces an $H_{0}$ value $>3 \sigma$ lower than R16 provides strong evidence that the $H_{0}$ discrepancy cannot be explained solely by a systematic specific to the Planck data. On the other hand, using the differenceof-covariance method described in Section 4.1 of Planck Collaboration et al. (2016), the shift in $H_{0}$ from adding the BAO to the $\ell>800$ Planck temperature power spectrum is larger than expected at the 2.2 and $2.8 \sigma$ level for the $\tau=0.07 \pm 0.02$ and $0.055 \pm 0.009$ priors, respectively.

The CMB + Ly $\alpha$ BAO fits yield higher values of $H_{0}$ than the CMB alone, without significantly smaller uncertainties. This reflects the tension between the CMB and Ly $\alpha$ BAO discussed in earlier work (e.g., Delubac et al. 2015: Planck Collaboration et al. 2016). In a joint tit with all the $\mathrm{BAO}$ data the $\mathrm{Ly} \alpha$ measurements lack the constraining power to overcome the galaxy BAO, and consequently our results are fairly insensitive to whether the Ly $\alpha$ are included along with the galaxy BAO or not. The interaction between the galaxy and Ly $\alpha$ BAO constraints is discussed further in Section 3.3. 
Table 2

Constraints on $H_{0}$ in the $\Lambda$ CDM model from the CMB alone and from combining CMB with BAO data, with the significance of the difference from the distance ladder measurement (73.24 \pm 1.74 ; Riess et al. 2016) in parenthesis, assuming uncorrelated Gaussian errors (all values in $\mathrm{km} \mathrm{s}^{-1} \mathrm{Mpc}^{-1}$ )

\begin{tabular}{|c|c|c|c|c|c|}
\hline CMB dataset & Large-scale likelihood $^{\mathrm{a}}$ & Power spectrum likelihoods ${ }^{\mathrm{b}}$ & $H_{0}$ (CMB only) & BAO datac & $H_{0}(\mathrm{CMB}+\mathrm{BAO})$ \\
\hline WMAP 9-year & " & " & " & Ly $\alpha$ & $71.01 \pm 2.10(0.8 \sigma)$ \\
\hline \multirow[t]{4}{*}{ ACTPol Two-Season } & $\tau=0.07 \pm 0.02$ & $\mathrm{TT}, \mathrm{TE}, \mathrm{EE}, \phi \phi$ & $67.12 \pm 2.67(1.9 \sigma)$ & $\mathrm{gal}+\mathrm{Ly} \alpha$ & $67.23 \pm 0.80(3.1 \sigma)$ \\
\hline & " & " & " & gal & $66.94 \pm 0.77(3.3 \sigma)$ \\
\hline & $"$ & " & & Ly $\alpha$ & $69.59 \pm 2.61(1.3 \sigma)$ \\
\hline & 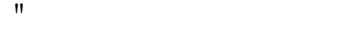 & $\mathrm{TT}, \mathrm{TE}, \mathrm{EE}$ & $67.60 \pm 3.56(1.4 \sigma)$ & $\mathrm{gal}+\mathrm{Ly} \alpha$ & $67.29 \pm 0.83(3.1 \sigma)$ \\
\hline \multirow[t]{5}{*}{ SPT-SZ } & $\tau=0.07 \pm 0.02$ & $\mathrm{TT}, \phi \phi$ & $71.38 \pm 3.09(0.5 \sigma)$ & gal + Ly $\alpha$ & $68.52 \pm 0.90(2.4 \sigma)$ \\
\hline & " & & " & gal & $68.25 \pm 0.91(2.5 \sigma)$ \\
\hline & $"$ & $"$ & & Ly $\alpha$ & $73.74 \pm 2.84(0.2 \sigma)$ \\
\hline & $"$ & $\mathrm{TT}$ & $73.20 \pm 3.54(0.0 \sigma)$ & gal + Ly $\alpha$ & $68.49 \pm 0.92(2.4 \sigma)$ \\
\hline & $\tau=0.055 \pm 0.009$ & $\mathrm{TT}, \phi \phi$ & $70.67 \pm 3.06(0.7 \sigma)$ & $\operatorname{gal}+\operatorname{Ly} \alpha$ & $68.46 \pm 0.88(2.5 \sigma)$ \\
\hline \multirow{6}{*}{ Planck } & $\tau=0.055 \pm 0.009$, low $\mathrm{l}^{\mathrm{e}}$ & plikHM_TT 2015 & $66.88 \pm 0.91(3.2 \sigma)$ & $\mathrm{gal}+\mathrm{Ly} \alpha$ & $67.72 \pm 0.54(3.0 \sigma)$ \\
\hline & $\tau=0.055 \pm 0.009$, lowl & plikHM_TTTEEE 2015 & $66.93 \pm 0.62(3.4 \sigma)$ & $\operatorname{gal}+\operatorname{Ly} \alpha$ & $67.53 \pm 0.45(3.2 \sigma)$ \\
\hline & $\tau=0.07 \pm 0.02$, lowl & plikHM_TT $\ell<800$ & $70.08 \pm 1.96(1.2 \sigma)$ & $\mathrm{gal}+\mathrm{Ly} \alpha$ & $68.34 \pm 0.67(2.6 \sigma)$ \\
\hline & $\tau=0.055 \pm 0.009$, low 1 & plikHM_TT $\ell<800$ & $69.78 \pm 1.86(1.4 \sigma)$ & $\operatorname{gal}+\operatorname{Ly} \alpha$ & $68.29 \pm 0.66(2.7 \sigma)$ \\
\hline & $\tau=0.07 \pm 0.02$ & plikHM_TT $\ell>800$ & $65.12 \pm 1.45(3.6 \sigma)$ & $\mathrm{gal}+\mathrm{Ly} \alpha$ & $67.91 \pm 0.66(2.9 \sigma)$ \\
\hline & $\tau=0.055 \pm 0.009$ & plikHM_TT $\ell>800$ & $64.30 \pm 1.31(4.1 \sigma)$ & $\mathrm{gal}+\mathrm{Ly} \alpha$ & $67.55 \pm 0.62(3.1 \sigma)$ \\
\hline
\end{tabular}

${ }^{a}$ Pixel-based and other likelihoods used at multipoles $\ell \lesssim 30$. For some fits, particularly with the ACTPol and SPT experiments that do not probe these scales, we indicate the Gaussian prior adopted on $\tau$ instead.

${ }^{\mathrm{b}}$ Temperature, E-mode polarization, temperature-polarization cross-spectrum and lensing potential power spectra are denoted TT, EE, TE, and $\phi \phi$, respectively.

c'gal' refers to galaxy BAO; 'Ly $\alpha$ ' refers to Lyman- $\alpha$ forest and QSO $\times$ Ly $\alpha$ BAO (see Table 1).

$\mathrm{d}$ lowTEB is the combined temperature-plus-polarization Planck likelihood for $\ell<30$.

eSince the Planck Collaboration Int. LI (2016) low-multipole polarization likelihood is not publicly available we approximate its inclusion with a prior $\tau=0.055 \pm 0.009$, which produces constraints in very good agreement with their Table 8. lowl is the Planck temperature-only likelihood for $\ell<30$ (no polarization).

We note that the SPT values in Table 2 differ from the value of $75.0 \pm 3.5 \mathrm{~km} \mathrm{~s}^{-1} \mathrm{Mpc}^{-1}$ quoted in Table 3 of the original SPT analysis by Story et al. (2013). This difference is driven by three effects: (i) the inclusion of the SPT lensing $\phi \phi$ power spectrum measurement from van Engelen et al. (2012) in some of our fits, (ii) the difference in $\tau$ prior: we used $0.07 \pm 0.02$ or $0.055 \pm 0.009$, while Story et al. (2013) used $0.088 \pm 0.015$, and (iii) different CosmoMC versions or fitting options, including the fact that we set the total neutrino mass to $0.06 \mathrm{eV}$ in our fits, while Story et al. (2013) assumed massless neutrinos, which leads to $\mathrm{a} \sim 0.2 \sigma$ shift in $H_{0}$. We have verified that if we use the Story et al. (2013) assumptions we reproduce their $75.0 \pm 3.5$ constraint. Aylor et al. (2017) recently derived parameters from SPT with an updated Planck-based calibration and improved likelihood, however the shift they report in $H_{0}$ is small and would not meaningfully affect our results.

\subsection{Angle-averaged versus anisotropic BAO}

Bennett et al. (2014; hereafter B14) used pre-Planck CMB data along with BAO measurements available at the time (6dFGS, BOSS DR11, including the Ly $\alpha$ but not $\mathrm{QSO} \times \mathrm{Ly} \alpha$ cross-correlation; we refer to these data as BAO14) to constrain

$$
\begin{gathered}
H_{0}=69.3 \pm 0.7 \mathrm{~km} \mathrm{~s}^{-1} \mathrm{Mpc}^{-1} \\
(\text { WMAP }+\mathrm{ACT}+\mathrm{SPT}+\mathrm{BAO} 14) .
\end{gathered}
$$

This value is noticeably higher than the $\mathrm{CMB}+\mathrm{BAO}$ values reported in Table 2 . To make a more direct comparison we performed an updated fit using WMAP, ACTPol, $\mathrm{SPT}$, and the latest BAO data, and find

$$
\begin{aligned}
& H_{0}=68.34 \pm 0.61 \mathrm{~km} \mathrm{~s}^{-1} \mathrm{Mpc}^{-1} \\
& (W M A P+\mathrm{ACTPol}+\mathrm{SPT}+\mathrm{BAO}) .
\end{aligned}
$$

The difference in these values appears large given the overlap in data sets used and so we investigated this difference in detail. We found that the downward shift in our current fits is due to a combination of several effects:

(i) The biggest difference comes from using the transverse and line-of-sight BOSS BAO scale measurements now available separately rather than the angle-averaged $D_{V}(z) / r_{d}$ used in B14. Using the BOSS DR11 CMASS anisotropic BAO instead of the BOSS DR11 CMASS angle-averaged BAO shifts the WMAP $+\mathrm{ACT}+\mathrm{SPT}+\mathrm{BAO} 14 H_{0}$ constraint downwards by $0.61 \mathrm{~km} \mathrm{~s}^{-1} \mathrm{Mpc}^{-1}$, a shift 

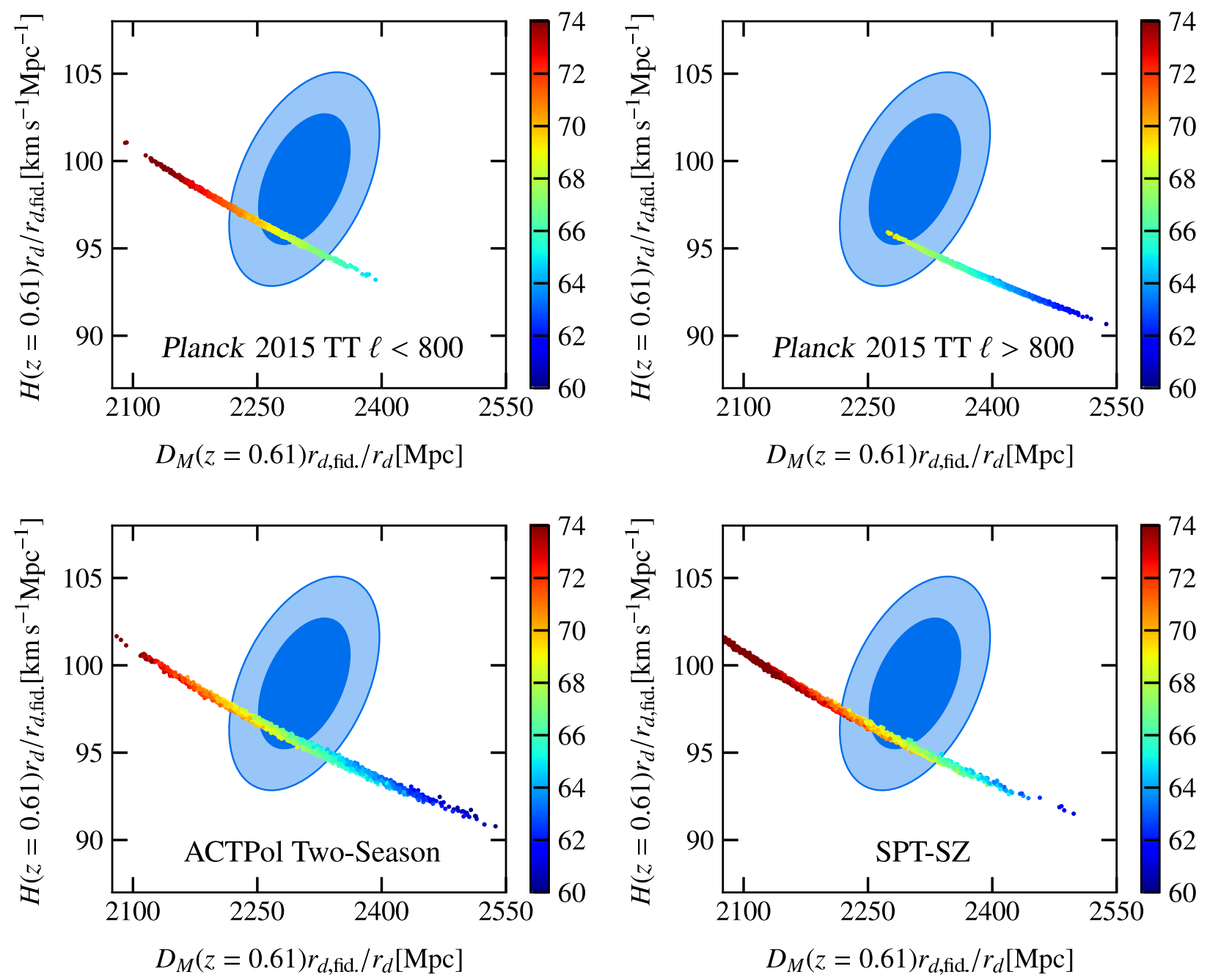

Figure 1. Including BAO data substantially tightens CMB constraints on $H_{0}$. The observables corresponding to the transverse and

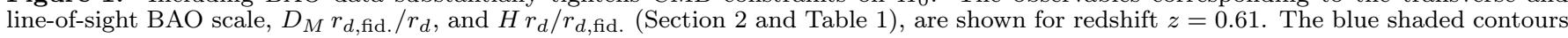
are the measurements from the final BOSS DR12 analysis (Alam et al. 2017). The different panels contain predictions from different, essentially independent, CMB measurements assuming a flat $\Lambda$ CDM model, with MCMC samples color-coded by $H_{0}$ in km s${ }^{-1} \mathrm{Mpc}^{-1}$. The same $\tau=0.07+0.02$ prior is used in each case. The addition of the BAO tightens the $H_{0}$ constraint by more than a factor of three in the case of ACTPol or SPT data (Table 2). When combined with any current CMB data set the galaxy BAO disfavor the values of $H_{0}$ preferred by the distance ladder $\left(73.24 \pm 1.74 \mathrm{~km} \mathrm{~s}^{-1} \mathrm{Mpc}^{-1}\right.$; Riess et al. 2016) at moderate to high significance. The lower values preferred by the high-multipole Planck data (the constraint from the samples shown in the top-right panel is $65.12 \pm 1.45 \mathrm{~km} \mathrm{~s}^{-1} \mathrm{Mpc}^{-1}$ ) are also disfavored.

comparable to the total uncertainty. This is discussed in more detail below.

(ii) A smaller shift of around $0.2 \mathrm{~km} \mathrm{~s}^{-1} \mathrm{Mpc}^{-1}$ is due to different likelihood codes. We find $H_{0}=69.07 \pm 0.70 \mathrm{~km} \mathrm{~s}^{-1} \mathrm{Mpc}^{-1}$ using WMAP $9+\mathrm{ACT}+\mathrm{SPT}+\mathrm{BAO} 14$. Our results were obtained with the November 2016 versions of $\mathrm{CAME}^{11}$ and CosmoMC, while a different MCMC code was used in B14. Furthermore, our implementation of the DR11 Ly $\alpha$ BAO constraint uses the $\chi^{2}$ look-up tables provided by BOSS ${ }^{12}$, whereas B14 constructed a likelihood directly from values reported by Delubac et al. (2015).

11 http://camb.info/

12 http://darkmatter.ps.uci.edu/baofit/ (iii) The ACTPol data have a stronger downward pull on $H_{0}$ than ACT. Both ACT and ACTPol prefer a lower $H_{0}$ value than WMAP alone (Sievers et al. 2013 Louis et al. 2017). The SPT data prefer a higher $H_{0}$ value than WMAP, and this preference wins out in the combination with ACT. With ACTPol, however, the downward pull is stronger, and the resulting constraint shifts downwards from $69.98 \pm 1.58(W M A P 9+\mathrm{ACT}+\mathrm{SPT})$ to $69.08 \pm$ $1.37 \mathrm{~km} \mathrm{~s}^{-1} \mathrm{Mpc}^{-1}$ (WMAP+ACTPol+SPT). In combination with the $\mathrm{BAO}$ the impact of using ACTPol instead of ACT is subdominant to the choice of BAO constraints.

(iv) The SDSS MGS BAO constraint at $z_{\text {eff }}=0.15$ was not used by B14. While the MGS measurement has lower precision than BOSS (4\% compared to 

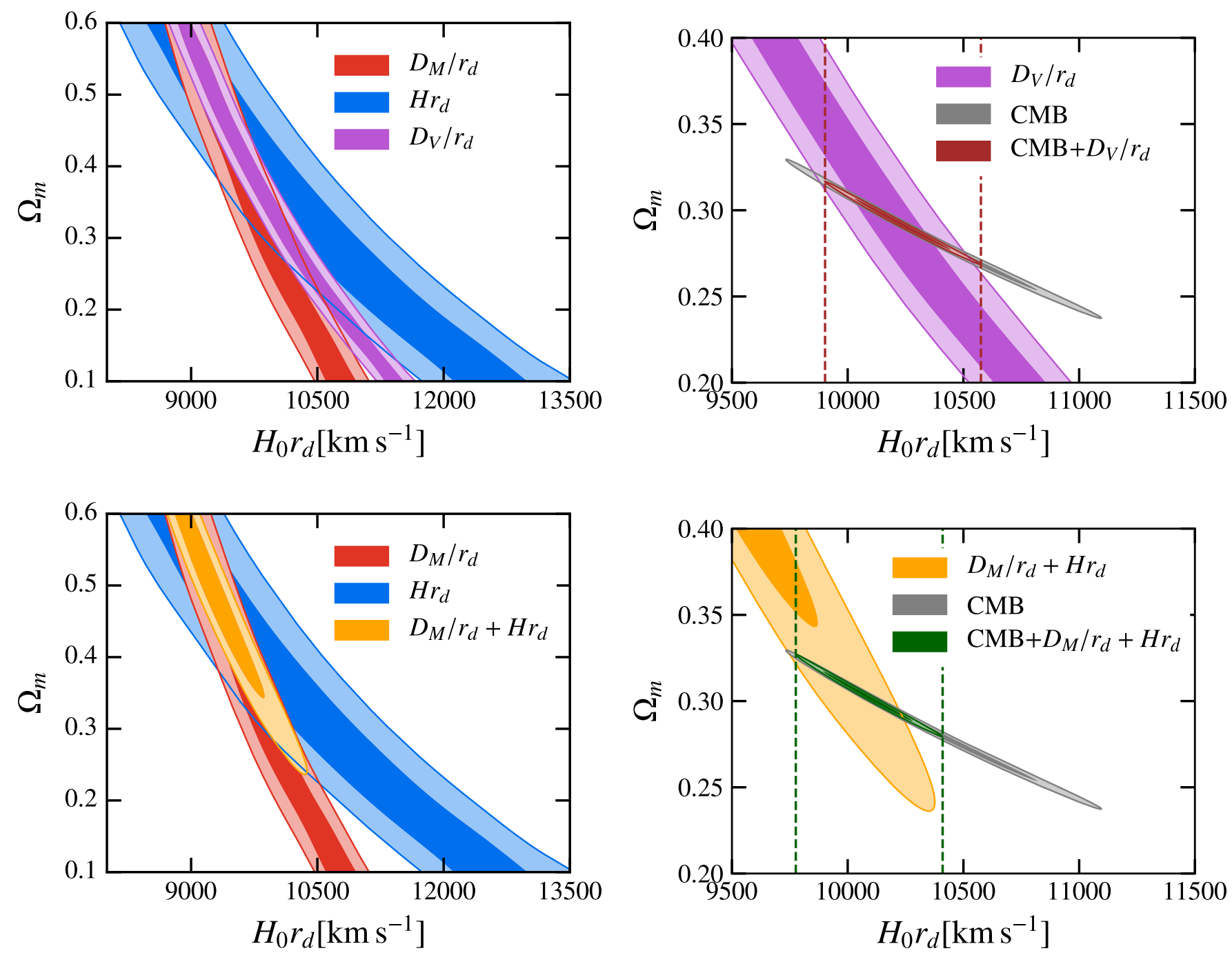

Figure 2. Use of the angle-averaged $\mathrm{BAO}$ constraint, $D_{V}(z) / r_{d}$, instead of the full anisotropic information, $D_{M}(z) / r_{d}$ plus $H(z) r_{d}$, can impact determination of $H_{0}$ from combined CMB $+\mathrm{BAO}$ fits. The upper left panel shows constraints from the same BOSS CMASS DR11 galaxy sample at $z_{\text {eff }}=0.57$ (Anderson et al. 2014) but different BAO measures - transverse $\left(D_{M} / r_{d}\right)$, line-of-sight $\left(H r_{d}\right)$, and angle-averaged $\left(D_{V} / r_{d}\right.$, see Section 2.1). The lower left panel shows the anisotropic constraint from combining $D_{M}(z) / r_{d}$ and $H(z) r_{d}$. While there is significant overlap between the angle-averaged and anisotropic contours, the angle-averaged contour extends to lower values of $\Omega_{m}$, which are not allowed by the anisotropic constraint. The upper right and lower right panels show the effect of adding the BAO information to CMB data (we show the same data sets used by Bennett et al. 2014. WMAP + ACT + SPT). The use of the angle-averaged $D_{V}(z) / r_{d}$ constraint diminishes the downward pull on $H_{0} r_{d}$, and also $H_{0}$, trom the BAO. The vertical dashed lines correspond to the bounds of the contours containing $95 \%$ of the $\mathrm{CMB}+\mathrm{BAO}$ MCMC samples.

around $1 \%$ ), it also has a stronger preference for lower $H_{0}$ in conjunction with the $\mathrm{CMB}$ data.

Why does the choice of anisotropic or angle-averaged BOSS CMASS BAO make such a large difference given the same galaxy sample is used for each? In the flat $\Lambda \mathrm{CDM}$ model, all the information from any BAO measurement is contained in contours in the two-dimensional $\Omega_{m}-H_{0} r_{d}$ space (Addison et al. 2013). The relative late-time expansion history is determined by $\Omega_{m}$, with $\Omega_{\Lambda}$ determined implicitly by the flatness constraint. The impact of radiation on the late-time expansion is small enough compared to the precision of current BAO measurements that uncertainties in the CMB temperature, which constrains the physical density $\Omega_{r} h^{2}$, or in converting to the fractional density, $\Omega_{r}$, can be neglected. The combination $H_{0} r_{d}$ provides an overall normalization factor and reflects the fact that the absolute length of the sound horizon, and a change in the normalization of the expansion rate, are completely degenerate when only fitting to measurements of the BAO scale.

The upper left and lower left panels of Figure 2 shows constraints in the $\Omega_{m}-H_{0} r_{d}$ plane for the DR11 BOSS CMASS sample at $z_{\text {eff }}=0.57$ (Anderson et al. 2014). We show the transverse $\left(D_{M} / r_{d}\right)$ and line-of-sight $\left(H r_{d}\right)$ contours separately, as well as the contour from combining both, and the angle-averaged $D_{V}(z) / r_{d}$ contour. While there is substantial overlap between the combined anisotropic contour and the $D_{V}(z) / r_{d}$ contour, a portion of the parameter space is allowed by $D_{V}(z) / r_{d}$ but ruled out by the combined anisotropic measurements. This portion is relevant when the $\mathrm{BAO}$ and $\mathrm{CMB}$ are combined, as shown in the upper right and lower right panels 
of Figure 2, with the anisotropic $D_{M}(z) / r_{d}+H(z) r_{d}$ constraint pulling down more strongly on $H_{0} r_{d}$, and hence $H_{0}$, since $H_{0}$ and $r_{d}$ are only partially degenerate in the CMB. The same effect is apparent in Figure 8 of Cuesta et al. (2016).

We conclude that the shift in $H_{0}$ from using the angleaveraged $D_{V}(z) / r_{d}$ instead of the full anisotropic BAO information is not caused by an inconsistency in the BAO measurements, but instead due to the compression of information inherent to $D_{V}(z) / r_{d}$. It is therefore preferable to use the anisotropic constraints where possible.

\subsection{Constraints from the BAO scale alone}

We now consider constraints from the BAO data without the strong additional constraining power of the CMB anisotropy measurements. As discussed above, in the flat $\Lambda \mathrm{CDM}$ model, BAO measurements provide contours in the $\Omega_{m}-H_{0} r_{d}$ plane. Combining the galaxy and Ly $\alpha$ BAO provides a tight constraint on $\Omega_{m}$ from the latetime expansion history, even when marginalizing over the normalization $H_{0} r_{d}$. For the BAO listed in Table 1 we find constraints of

$$
\begin{aligned}
\Omega_{m} & =0.292 \pm 0.020 \\
H_{0} r_{d} & =(10119 \pm 138) \mathrm{km} \mathrm{s}^{-1} .
\end{aligned}
$$

The left panel of Figure 3 shows constraints from the galaxy and Ly $\alpha \mathrm{BAO}$ in the $\Omega_{m}-H_{0} r_{d}$ plane. The orientation of these contours can be approximately understood from considering the redshift dependence of $H(z)$. Similar arguments hold for $D_{A}(z)$. At the Ly $\alpha$ $\mathrm{BAO}$ redshifts the universe is matter dominated, and $H(z) \simeq H_{0} \Omega_{m}^{1 / 2}(1+z)^{3 / 2}$, so that $H(z) r_{d}$ constraints produce contours along the direction with $H_{0} r_{d} \cdot \Omega_{m}^{1 / 2}$ roughly constant. At lower redshifts, where dark energy becomes dominant, $H(z)$ depends less strongly on $\Omega_{m}$, leading to the galaxy BAO contour being oriented more along the direction of the $\mathrm{y}$-axis in Figure $3^{13}$ There is little overlap between the galaxy and Ly $\alpha$ contours. To quantify this difference, we consider the test described in Section 4.1 of Hou et al. (2014). We calculate $\Delta \chi^{2}=\chi_{X+Y}^{2}-\chi_{X}^{2}-\chi_{Y}^{2}$, where in this case $X$ and $Y$ are the galaxy and $\operatorname{Ly} \alpha$ BAO data, respectively, $\chi_{X+Y}^{2}$ denotes the best-fit $\chi^{2}$ from the joint fit, and $\chi_{X}^{2}$ and $\chi_{Y}^{2}$ are the best-fit $\chi^{2}$ from the fits to just the galaxy or just the Ly $\alpha$ data. For Gaussian-distributed data 14 if $X$ and $Y$ are independent and $\Lambda$ CDM is the correct model then $\Delta \chi^{2}$ is drawn from a $\chi^{2}$ distribution with $N_{\Delta \chi^{2}}=N_{X+Y}-N_{X}-N_{Y}$ degrees of freedom (dof). We find

$$
\begin{aligned}
\chi_{\text {gal }}^{2} & =2.98 & N_{\text {gal }} & =8-2=6 \\
\chi_{\text {Ly } \alpha}^{2} & =0.92 & N_{\text {Ly } \alpha} & =4-2=2 \\
\chi_{\text {gal }+ \text { Ly } \alpha}^{2} & =13.63 & N_{\text {gal }+ \text { Ly } \alpha} & =12-2=10 \\
\Delta \chi^{2} & =9.73 & N_{\Delta \chi^{2}} & =10-6-2=2
\end{aligned}
$$

The probability to exceed (PTE) for $\chi^{2}=9.73$ with $N_{\text {dof }}=2$ is $7.71 \times 10^{-3}$, which corresponds to a $2.4 \sigma$

13 If BAO measurements at $z=0$ were possible they would produce exactly vertical contours in Figure 3.

14 This is a reasonable approximation when the Ly $\alpha$ and $\mathrm{QSO} \times \operatorname{Ly} \alpha$ BAO are combined (Delubac et al. 2015). disagreement. This is comparable to the $2.5 \sigma$ tension reported between the Ly $\alpha \mathrm{BAO}$ and Planck measurements by Delubac et al. (2015). As discussed by Aubourg et al. (2015), modifying the cosmological model to improve three-way agreement between $\mathrm{CMB}$, galaxy BAO, and $\mathrm{Ly} \alpha \mathrm{BAO}$ appears difficult. Here we note that the combined contour in Figure 3 lies at the intersection of the main degeneracy directions determined by the redshift coverage of the galaxy and Ly $\alpha$ measurements. If future data shift the galaxy or Ly $\alpha \mathrm{BAO}$ constraints along these degeneracy lines (as opposed to perpendicular to them) the main result would be to change the quality of the combined fit rather than changing the parameter values. We further note that the matter density reported in (4) is in agreement with the value of $0.295 \pm 0.034$ from a joint analysis of type Ia $\mathrm{SNe}$ from several surveys covering $0<z<1$, completely independent of LSS clustering (Betoule et al.2014). This is illustrated in the right panel of Figure 3, which shows a comparison of BAO, WMAP 9-year, Planck 2016 15 and SNe constraints on $\Omega_{m}$ for the flat $\Lambda$ CDM model.

\subsection{Constraining $H_{0}$ with $B A O$ plus deuterium abundance in $\Lambda \mathrm{CDM}$}

Obtaining a constraint on $H_{0}$ from the BAO requires adding information to break the $H_{0}-r_{d}$ degeneracy. One way to do this is to add a constraint on the baryon density (e.g., Addison et al. 2013; Aubourg et al. 2015; Wang et al. 2017). We assume that the photon energy density, or, equivalently, the CMB mean temperature, is also known. The CMB temperature was measured precisely by $C O B E /$ FIRAS (Fixsen et al. 1996, Fixsen 2009) and we view this result as independent of the CMB anisotropy measurements performed by more recent experiments. Note that while the $H_{0}$ in the $H(z)$ in the denominator of (1) cancels in the $H_{0} r_{d}$ product, some residual $H_{0}$ dependence still exists because the decoupling redshift and the sound speed depend on the physical matter and radiation densities, $\Omega_{m} h^{2}$ and $\Omega_{r} h^{2}$, respectively, while the expansion rate $H(z)$ depends on the fractional densities $\Omega_{m}$ and $\Omega_{r}$.

In the BAO fit with an external baryon density prior, $\Omega_{m}$ performs double duty. It not only goes into determining the late-time expansion $\left(D_{M}\right.$ and $H$ at the BAO survey redshifts) but also controls the expansion history in the early universe prior to baryons decoupling from photons, since the photon and neutrino properties (with $\left.N_{\text {eff }}=3.046\right)$ are held fixed. As well as providing an indirect $H_{0}$ constraint, the $\mathrm{BAO}+\Omega_{b} h^{2}$ fit also serves as something of a self-consistency test of early and late-time expansion.

The most precise constraints on $\Omega_{b} h^{2}$ independent of the CMB power spectrum come from estimates of the primordial deuterium abundance. In standard Big Bang nucleosynthesis $(\mathrm{BBN})$, the abundance of light nuclei is determined by a single parameter, the baryon-to-photon ratio $\eta$ (see recent review by Cyburt et al. 2016, and references therein). Taking the photon number density as fixed from the CMB temperature, a measurement of the primordial deuterium abundance in conjunction with

15 We refer to the combination of the 2015 TT constraints with updated $\tau=0.055 \pm 0.009$ prior from Planck Collaboration Int. LI (2016) as 'Planck 2016'. 

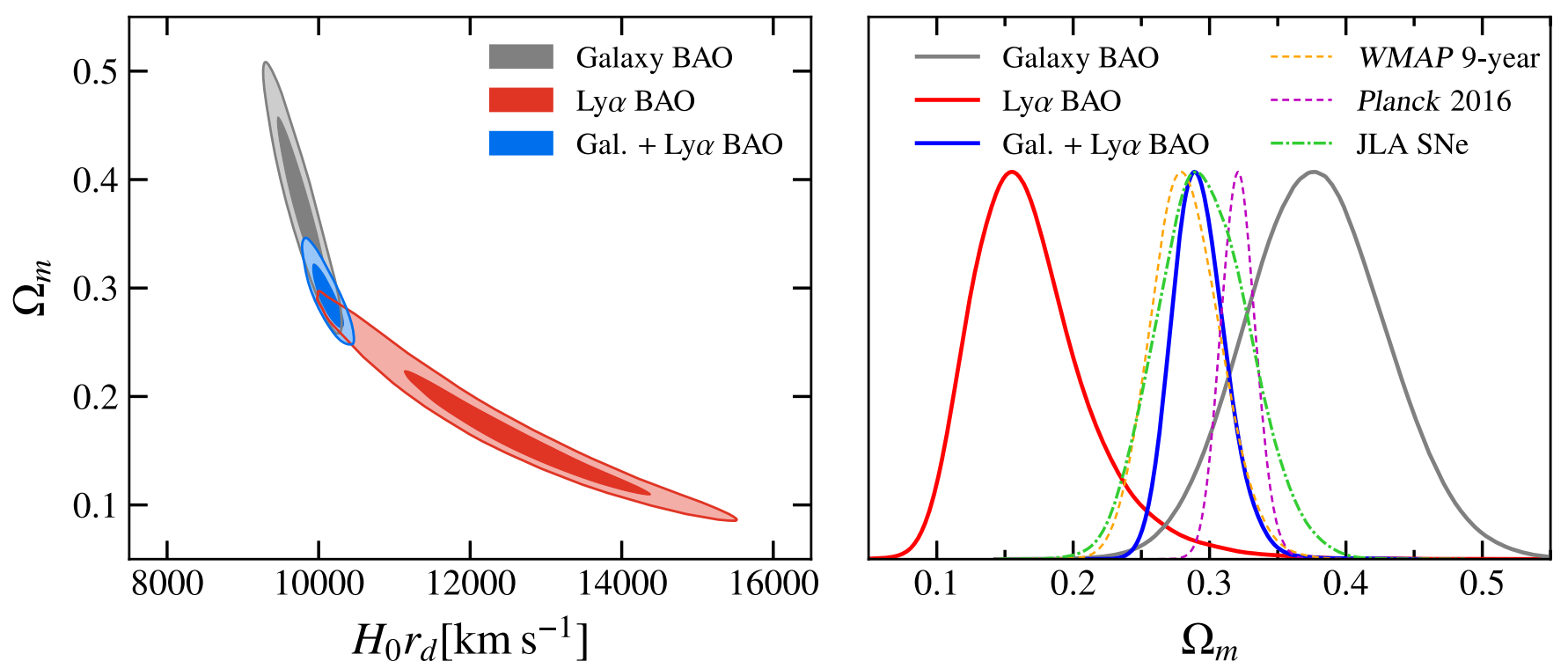

Figure 3. Left: Comparison of BAO-only constraints in the flat $\Lambda$ CDM model. Contours containing 68 and $95 \%$ of MCMC samples are shown for galaxy $\left(z_{\text {eff }} \leq 0.61\right)$ and Ly $\alpha$ forest $\left(z_{\text {eff }} \geq 2.3\right)$ BAO separately and in a joint fit using the BAO data listed in Table 1 . In flat $\Lambda$ CDM the late-time expansion rate is determined only by $\Omega_{m}$, with $H_{0} r_{d}$ acting as an overall expansion normalization. Right: Comparison of $\Omega_{m}$ constraints from BAO, CMB and SNe measurements. The SNe constraint is from the "joint light-curve analysis" (JLA) presented by Betoule et al. (2014). While the combined BAO fit produces a tight constraint $\Omega_{m}=0.293 \pm 0.020$, in agreement with the CMB and SNe determinations, there is a $2.4 \sigma$ tension between the galaxy and Ly $\alpha \mathrm{BAO}$, which individually prefer higher and lower values of $\Omega_{m}$, respectively.

Table 3

$\Lambda \mathrm{CDM}$ constraints from the $\mathrm{BAO}+\mathrm{D} / \mathrm{H}$ fits, using either the theoretical or empirical $d(p, \gamma)^{3} \mathrm{He}$ reaction rate, with CMB anisotropy constraints from WMAP and Planck included for comparison

\begin{tabular}{lcccc}
\hline Parameter & $\begin{array}{c}\mathrm{BAO}+\mathrm{D} / \mathrm{H} \\
\text { (theoretical) }\end{array}$ & $\begin{array}{c}\text { BAO }+\mathrm{D} / \mathrm{H} \\
\text { (empirical) }\end{array}$ & WMAP 9-year & Planck 2016 \\
\hline $100 \Omega_{b} h^{2}$ & $2.156 \pm 0.020$ & $2.257 \pm 0.034$ & $2.265 \pm 0.049$ & $2.215 \pm 0.021$ \\
$100 \Omega_{c} h^{2}$ & $10.94 \pm 1.20$ & $11.19 \pm 1.29$ & $11.37 \pm 0.46$ & $12.07 \pm 0.21$ \\
$100 \theta_{\mathrm{MC}}$ & $1.0292 \pm 0.0168$ & $1.0320 \pm 0.0173$ & $1.04025 \pm 0.00223$ & $1.04076 \pm 0.00047$ \\
$H_{0}\left[\mathrm{~km} \mathrm{~s}^{-1} \mathrm{Mpc}^{-1}\right]$ & $66.98 \pm 1.18$ & $67.81 \pm 1.25$ & $69.68 \pm 2.17$ & $66.89 \pm 0.90$ \\
$\Omega_{m}$ & $0.293 \pm 0.019$ & $0.293 \pm 0.020$ & $0.283 \pm 0.026$ & $0.321 \pm 0.013$ \\
$r_{d}[\mathrm{Mpc}]$ & $151.6 \pm 3.4$ & $149.2 \pm 3.6$ & $148.49 \pm 1.23$ & $147.16 \pm 0.48$ \\
\hline
\end{tabular}

knowledge of BBN physics provides a constraint on $\Omega_{b} h^{2}$. Precise estimates of the primordial deuterium abundance have been made in recent years using extremely metalpoor damped Lyman- $\alpha$ (DLA) systems along sight lines to high-redshift quasars (e.g., Pettini \& Cooke 2012; Cooke et al. 2014, 2016; Riemer-Sørensen et al. 2017). Cooke et al. (2016; hereafter C16) report

$$
10^{5} \mathrm{D}_{\mathrm{I}} / \mathrm{H}_{\mathrm{I}}=2.547 \pm 0.033
$$

by combining six such systems. The $d(p, \gamma)^{3}$ He reaction rate plays a key role in the conversion from $\mathrm{D} / \mathrm{H}$ to $\Omega_{b} h^{2}$. Using the theoretical calculation for this rate from Marcucci et al. (2016), C16 find

$$
\begin{array}{r}
100 \Omega_{b} h^{2}=2.156 \pm 0.020 \\
(\mathrm{D} / \mathrm{H}, \text { theoretical rate }),
\end{array}
$$

which is $>2 \sigma$ lower than the Planck value (assuming a standard $\Lambda$ CDM model throughout). Using instead an empirically derived $d(p, \gamma)^{3} \mathrm{He}$ rate, $\mathrm{C} 16$ find

$$
100 \Omega_{b} h^{2}=2.260 \pm 0.034
$$

(D/H, empirical rate), which has a larger uncertainty but is in better agreement with CMB-derived values. We performed fits to the galaxy plus Ly $\alpha \mathrm{BAO}$ data with the addition of each of the Gaussian priors on $\Omega_{b} h^{2}$ in (6) and (7) in turn. We show parameter constraints in Table 3 , including the WMAP 9-year and Planck 2016 CMB anisotropy constraints for comparison.

In the $\mathrm{BAO}+\mathrm{D} / \mathrm{H}$ fits, $\Omega_{b} h^{2}$ is driven solely by the $\mathrm{D} / \mathrm{H}$ prior, as expected, and $\Omega_{m}$ matches the BAO-only value. While the choice of the $d(p, \gamma)^{3} \mathrm{He}$ reaction rate significantly impacts the value of $\Omega_{b} h^{2}$, it has a reduced impact on the inferred $H_{0}$, because $r_{d}$ only depends weakly on $\Omega_{b} h^{2}$ (Eisenstein \& Hu 1998. Addison et al. 2013). Specifically, replacing the theoretical rate with the empirical one shifts the center of the $\Omega_{b} h^{2}$ distribution by 5.2 times the original uncertainty, but shifts the $H_{0}$ distribution by only 0.7 times the original uncertainty. Our $\mathrm{BAO}+\mathrm{D} / \mathrm{H}$ results for $H_{0}$ are more robust to the choice of rate than one might expect from the $\Omega_{b} h^{2}$ difference.

The $H_{0}$ values listed in Table 3 from the $\mathrm{BAO}+\mathrm{D} / \mathrm{H}$ fits have uncertainties of around $1.8 \%$ and are 3.0 and $2.5 \sigma$ lower than the R16 distance ladder value of $73.24 \pm$ 


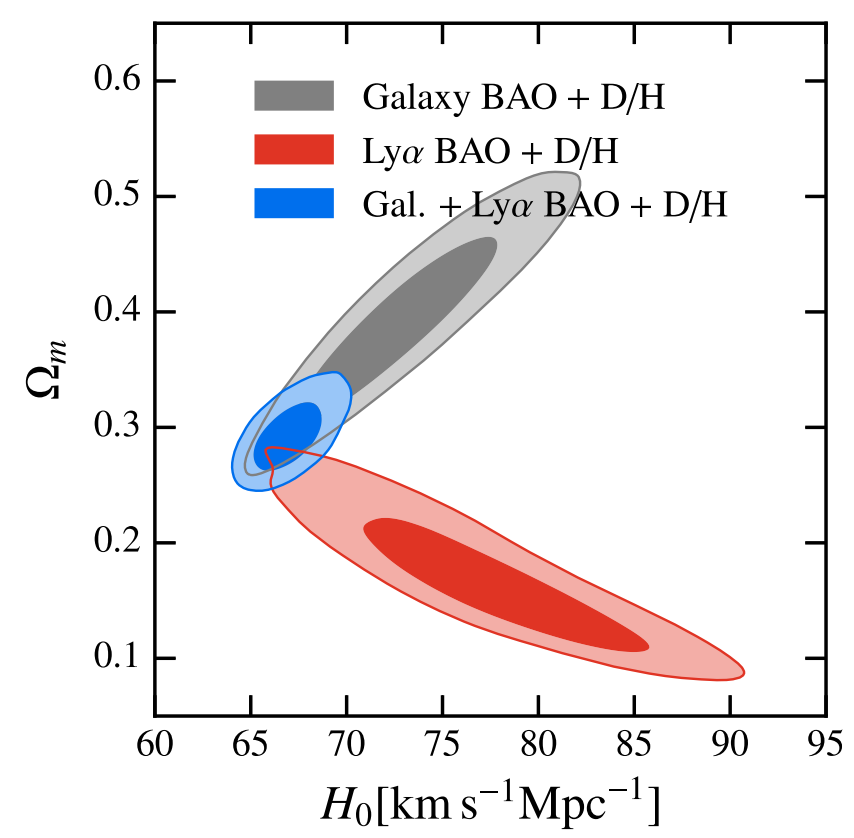

Figure 4. Adding an estimate of the baryon density, $\Omega_{b} h^{2}$, in this case from deuterium abundance $(\mathrm{D} / \mathrm{H})$ measurements, breaks the BAO $H_{0}-r_{d}$ degeneracy in $\Lambda$ CDM. The same contours are shown as in Figure 3, with the addition of a Gaussian prior $100 \Omega_{b} h^{2}=2.156 \pm 0.020$ (Cooke et al. 2016). In contrast to Figure 3 , here $\Omega_{m}$ determines both the early time expansion, including the absolute sound horizon, $r_{d}$, as well as the late-time expansion history. The radiation density is fixed from COBE/FIRAS CMB mean temperature measurements. The combined $\mathrm{BAO}+\mathrm{D} / \mathrm{H}$ constraint, $H_{0}=66.98 \pm 1.18 \mathrm{~km} \mathrm{~s}^{-1} \mathrm{Mpc}^{-1}$ is $3.0 \sigma$ lower than the Riess et al. (2016) distance ladder determination and is independent of CMB anisotropy data.

$1.74 \mathrm{~km} \mathrm{~s}^{-1} \mathrm{Mpc}^{-1}$ for the theoretical and empirical $d(p, \gamma)^{3} \mathrm{He}$ rates, respectively. The combination of precise $\mathrm{BAO}$ and $\mathrm{D} / \mathrm{H}$ measurements enables determinations of $H_{0}$ within the context of the flat $\Lambda \mathrm{CDM}$ model that are almost 50\% tighter than the distance ladder measurement, and lower at moderate to strong significance. We emphasize that these constraints are completely independent of CMB anisotropy measurements.

Constraints in the $\Omega_{m}-H_{0}$ plane for the $\mathrm{BAO}+\mathrm{D} / \mathrm{H}$ fits with the theoretical $d(p, \gamma)^{3} \mathrm{He}$ rate are shown in Figure 4. We show results from the galaxy and Ly $\alpha$ BAO separately and together, as before. Tension between the galaxy and $\mathrm{Ly} \alpha \mathrm{BAO}$ is again apparent. Adding $\mathrm{D} / \mathrm{H}$ to these data separately favors higher values of $H_{0}$, and it is only when galaxy and $\mathrm{Ly} \alpha \mathrm{BAO}$ are combined that $H_{0}$ is constrained to the values quoted in Table 3 .

The direction of the $\operatorname{Ly} \alpha \mathrm{BAO}$ contour is roughly the same in the left panel of Figure 3 and in Figure 4, while that of the galaxy BAO contour changes. This behavior can be understood by considering how $r_{d}$ depends on $\Omega_{m}$ and $H_{0}$. For a given value of $\Omega_{b} h^{2}, r_{d}$ depends approximately on the combination $H_{0} \cdot \Omega_{m}^{1 / 2}$ (equation 26 of Eisenstein \& Hu 1998). This is the same dependence as $H(z)$ at the Ly $\alpha$ redshifts (Section 3.3) and is related to the fact that the universe is largely matter dominated in both cases. The dependence of $H(z)$ on $\Omega_{m}$ at the galaxy BAO redshifts is weaker, and the direction of the galaxy BAO contour in Figure 4 is approximately deter- mined by requiring $H_{0} r_{d}$ to be roughly constant as $\Omega_{m}$ varies. This produces a positive correlation between $H_{0}$ and $\Omega_{m}$ because $r_{d}$ decreases as $H_{0} \Omega_{m}^{1 / 2}$ increases.

For the $\mathrm{BAO}+\mathrm{D} / \mathrm{H}$ fits, we ran CosmoMC as one would when fitting to the CMB: the fitted parameters are $\Omega_{b} h^{2}$, the physical cold dark matter density, $\Omega_{c} h^{2}$, and the angular sound horizon, $\theta_{\mathrm{MC}}$, and $H_{0}, \Omega_{m}$, and $r_{d}$ are derived from these three. Since the $\mathrm{BAO}+\mathrm{D} / \mathrm{H}$ data are insensitive to the amplitude and tilt of the primordial power spectrum, and the optical depth to reionization, these other $\Lambda \mathrm{CDM}$ parameters are held fixed. Consistent results were obtained using earlier $\mathrm{BAO}$ and $\mathrm{D} / \mathrm{H}$ data by Addison et al. (2013) and Aubourg et al. (2015). We note that Riemer-Sørensen \& Sem Jenssen (2017) recently obtained a tighter constraint on $\mathrm{D} / \mathrm{H}$ than we have used here by combining the DLAs used by C16 with a number of additional measurements. Using this tighter constraint would not impact our conclusions.

\subsection{BAO and light element abundance constraints with varying $N_{\text {eff }}$}

In the $\Lambda \mathrm{CDM}+N_{\text {eff }}$ model, there is a perfect degeneracy between $\Omega_{b} h^{2}$ and $N_{\text {eff }}$ from $\mathrm{D} / \mathrm{H}$ measurements (Fig. 6 of C16). Closed contours in the $\Omega_{b} h^{2}-N_{\text {eff }}$ plane can be obtained from combining estimates of the primordial $\mathrm{D} / \mathrm{H}$ and ${ }^{4} \mathrm{He}$ abundance (e.g., review by $\mathrm{Cy}-$ burt et al. 2016, and references therein). The primordial ${ }^{4} \mathrm{He}$ abundance is estimated from $\mathrm{He}$ and $\mathrm{H}$ emission lines in extragalactic HII regions. Obtaining accurate constraints is challenging due to dependence on environmental parameters such as temperature, electron density, and metallicity, which must be modeled. An important recent development is the use of the HeI line at $10830 \AA$ to help break modeling degeneracies (Izotov et al. 2014). The value of the primordial helium fraction reported by Izotov et al. (2014), $Y_{p}=0.2551 \pm 0.0022$, is, however, significantly higher than values found in some subsequent analyses of the same HII sample using different selection criteria and fitting methodology. For example, Aver et al. (2015) found $Y_{p}=0.2449 \pm 0.0040$, while Peimbert et al. (2016) found $Y_{p}=0.2446 \pm 0.0029$. The different $Y_{p}$ values lead to significantly different inferences for $N_{\text {eff }}$ when used in combination with $\mathrm{D} / \mathrm{H}$ or $\mathrm{CMB}$ power spectra measurements. Izotov et al. (2014) found evidence for additional neutrino species at $99 \%$ confidence, while, for instance, Cyburt et al. (2016) report $N_{\text {eff }}=2.85 \pm 0.28$, and Peimbert et al. (2016) found $N_{\text {eff }}=2.90 \pm 0.22$, consistent with the standard model value of 3.046 .

Current $\mathrm{D} / \mathrm{H}$ and ${ }^{4} \mathrm{He}$ constraints clearly have the precision to weigh in significantly on the question of whether allowing $N_{\text {eff }}>3$ is effective at resolving $\Lambda$ CDM tensions. Given the spread in $Y_{p}$ values discussed above, and the impact of the choice of $d(p, \gamma)^{3} \mathrm{He}$ rate when $N_{\text {eff }}$ is allowed to vary (Section 5.2 of C16), we do not present a full set of results including BAO and light element abundance data for $\Lambda \mathrm{CDM}+N_{\text {eff }}$. Instead we note that combining BAO measurements with $\mathrm{D} / \mathrm{H}$ and ${ }^{4} \mathrm{He}$ constraints on $N_{\text {eff }}$ that are consistent with the standard model value would produce $H_{0}$ values consistent with the values in Table 3 , although with larger uncertainties, while higher values of $N_{\text {eff }}$ would produce a higher $H_{0}$, improving agreement with the distance ladder. The BAO measurements, being only sensitive to $H_{0} r_{d}$, and not to 
$H_{0}$ or $N_{\text {eff }}$ directly, are unable to discriminate between these possibilities.

\section{DISCUSSION}

We have presented evidence for a lower $H_{0}$ value than measured by the local distance ladder that is independent of Planck, both from combining BAO with other CMB datasets (WMAP, ACTPol and SPT), and from joint fits to $\mathrm{BAO}$ and $\mathrm{D} / \mathrm{H}$ measurements, within the context of the standard $\Lambda \mathrm{CDM}$ model. In light of this analysis it is clear that the $H_{0}$ tension cannot be resolved solely through a systematic error specific to the Planck data. It should be noted, however, that it is not simply a case of having a 'high' $H_{0}$ from the distance ladder, and a 'low' $H_{0}$ from Planck and the joint BAO fits. The high-multipole Planck temperature data prefer $H_{0}$ values that are even lower than the $\mathrm{CMB}+\mathrm{BAO}$ or $\mathrm{BAO}+\mathrm{D} / \mathrm{H}$ values (bottom two rows of Table 2 and top right panel of Fig. 1). Restricting the Planck temperature power spectrum to multipoles $\ell>800$ produces

$$
\begin{aligned}
& H_{0}=65.12 \pm 1.45 \mathrm{~km} \mathrm{~s}^{-1} \mathrm{Mpc}^{-1} \\
& (\text { Planck } 2015 \mathrm{TT} \ell>800, \tau=0.07 \pm 0.02)
\end{aligned}
$$

or

$$
\begin{aligned}
& H_{0}=64.30 \pm 1.31 \mathrm{~km} \mathrm{~s}^{-1} \mathrm{Mpc}^{-1} \\
& (\text { Planck } 2015 \mathrm{TT} \ell>800, \tau=0.055 \pm 0.009),
\end{aligned}
$$

depending on the choice of $\tau$ prior. These values are not only in strong tension with R16, but are in moderate tension with some of the $\mathrm{CMB}+\mathrm{BAO}$ values reported in $\mathrm{Ta}-$ ble 2. For example, for $\tau=0.055 \pm 0.009$, the SPT $+\mathrm{BAO}$ value is lower than R16 by $2.5 \sigma$, but the Planck $\ell>800$ value is $2.6 \sigma$ lower again than $\mathrm{SPT}+\mathrm{BAO}$. The shift in $H_{0}$ from adding the BAO to the $\ell>800$ Planck constraints is also larger than expected given the improvement in precision, as discussed in Section 3.1. Some $H_{0}$ tension remains even if we do not consider the distance ladder constraints. In fact, concordance cannot be achieved through the removal of any single data set (e.g., $\mathrm{BAO}, \mathrm{CMB}$, distance ladder, or $\mathrm{D} / \mathrm{H}$ ). This is part of the reason the $H_{0}$ discrepancy is challenging to resolve: a convincing solution must simultaneously address multiple avenues of disagreement.

A wide range of fits to expanded cosmological models, with various combinations of data, have been presented in recent years to try to reconcile $H_{0}$ and other parameter tensions. Our fits in this paper have been restricted to the standard flat $\Lambda \mathrm{CDM}$ model, partly because our results for expanded models would be similar to those already presented by Planck Collaboration et al. (2016), Alam et al. (2017), Heavens et al. (2017), and others. The $\mathrm{BAO}, \mathrm{CMB}$, and light element abundance measurements have some common dependence on the early universe expansion history, which makes allowing freedom in, for example, $N_{\text {eff }}$, seem attractive. As discussed in Section 1, varying $N_{\text {eff }}$ does not sufficiently relieve tensions and is not statistically favored over standard $\Lambda \mathrm{CDM}$ for the current $\mathrm{BAO}, \mathrm{CMB}$, and distance ladder data. There are good prospects for tightening $N_{\text {eff }}$ constraints through improved measurements of the high- $\ell$ CMB damping tail in E-mode polarization (e.g., Abazajian et al. 2015, 2016). Future BAO data, for example from the Dark Energy Spectroscopic Instrument $\left(\right.$ DESS $\left.^{16}\right)$, Euclid ${ }^{17}$, and WFIRST ${ }^{18}$, will also provide significant improvements in precision over current measurements (for $\mathrm{BAO}+\Omega_{b} h^{2}$ forecasts for $H_{0}$, see Wang et al. 2017).

\section{CONCLUSIONS}

We have examined the role of BAO measurements in determining $H_{0}$. While the BAO data alone are unable to distinguish between a change in $H_{0}$ and a change in the absolute sound horizon at decoupling, $r_{d}$, this degeneracy is broken, and a precise $H_{0}$ value obtained, when $\mathrm{BAO}$ are combined with either CMB power spectra or deuterium abundance measurements. Overall we find convincing evidence for a lower $H_{0}$ in $\Lambda \mathrm{CDM}$ than obtained from the latest local distance ladder measurement (73.24 $1.74 \mathrm{~km} \mathrm{~s}^{-1} \mathrm{Mpc}^{-1}$; Riess et al. 2016) even without using data from Planck. The motivation and results of this study are summarized as follows:

(i) Tension at the $>3 \sigma$ level exists between determinations of $H_{0}$ from the distance ladder and the CMB anisotropy measurements from Planck, within the context of the standard flat $\Lambda \mathrm{CDM}$ model. Other tensions also exist, for example between Planck data and constraints on the growth of structure from some weak lensing surveys.

(ii) None of the cosmological modifications commonly proposed appear to provide a statistically compelling solution to these tensions, although some, such as allowing freedom in the number of effective relativistic species, $N_{\text {eff }}$, do reduce the $H_{0}$ disagreement.

(iii) Combining BAO measurements with $\mathrm{CMB}$ power spectrum data from WMAP, ACTPol, or SPT, produces $H_{0}$ values lower than the distance ladder by $2.4-3.1 \sigma$, independent of Planck (Table 2). The difference was less pronounced in some earlier analyses because of using the angle-averaged BOSS CMASS BAO measurement. The angle-averaged $D_{V}(z) / r_{d}$ constraint is a compression of information and allows a region of parameter space that is ruled out by the full anisotropic BAO constraints (Fig. 2). Adding the BAO improves the $H_{0}$ constraint from ACTPol or SPT by more than a factor of three, making their precision comparable to the Planck-only results.

(iv) Combining $\mathrm{BAO}$ data with primordial deuterium $(\mathrm{D} / \mathrm{H})$ abundance estimates from metal-poor DLA systems produces precise $H_{0}$ values lower than the distance ladder by $2.5-3.0 \sigma$, depending on assumptions about the $d(p, \gamma)^{3} \mathrm{He}$ reaction rate (e.g., $66.98 \pm 1.18 \mathrm{~km} \mathrm{~s}^{-1} \mathrm{Mpc}^{-1}$ for the theoretical rate, see Table 3). This result is independent of any CMB anisotropy measurement and relies only on the CMB mean temperature from $C O B E$ /FIRAS.

(v) The two previous results taken together indicate that it is not possible to resolve the $H_{0}$ disagree-

\footnotetext{
16 http://desi.lbl.gov/

17 https://www.euclid-ec.org/

18 https://wfirst.gsfc.nasa.gov/
} 
ment solely through some systematic error specific to the Planck data set.

(vi) The Planck high-multipole $(\ell>800)$ damping tail measurements prefer lower values of $H_{0}$ than the combined BAO fits, for example $65.12 \pm 1.45$ and $64.30 \pm 1.31 \mathrm{~km} \mathrm{~s}^{-1} \mathrm{Mpc}^{-1}$, for $\tau=0.07 \pm 0.02$ and $\tau=0.055 \pm 0.009$, respectively. The shift in $H_{0}$ from adding the BAO to these data is larger than expected at the 2.2 and $2.8 \sigma$ level for these $\tau$ priors. The $H_{0}$ disagreement is not as simple as the distance ladder value being 'high' and other constraints coming out 'low', and cannot be resolved through the removal of any single data set.

(vii) We note that a $2.4 \sigma$ tension exists between the galaxy $(z \leq 0.61)$ and $\operatorname{Ly} \alpha(z \geq 2.4) \mathrm{BAO}$, as previously discussed by Aubourg et al. (2015). The $\mathrm{BAO}+\mathrm{D} / \mathrm{H}$ constraints rely on combining these measurements and as such it is important to review their consistency with future data.

In recent years new precise measurements have led to multiple tensions, particularly in $H_{0}$, that are uncomfortably large to be explained by statistical scatter within the context of the standard $\Lambda$ CDM model. Whether this is the sign of new physics or underestimated uncertainties, or some combination of effects, remains unclear, and no straightforward explanation has yet presented itself. Near-term improvements in CMB, LSS, and distance ladder data are expected, however continuing to scrutinize existing measurements, as we have in this work, could also prove important in moving towards an eventual resolution.

This research was supported in part by NASA grants NNX15AJ57G, NNX16AF28G, and NNX17AF34G, and JPL grant 1563692, and by the Canadian Institute for Advanced Research (CIFAR). We acknowledge the use of the Legacy Archive for Microwave Background Data Analysis (LAMBDA), part of the High Energy Astrophysics Science Archive Center (HEASARC). HEASARC/LAMBDA is a service of the Astrophysics Science Division at the NASA Goddard Space Flight Center. This work was partly based on observations obtained with Planck (http://www.esa.int/Planck), an ESA science mission with instruments and contributions directly funded by ESA Member States, NASA, and Canada. Part of this research project was conducted using computational resources at the Maryland Advanced Research Computing Center (MARCC).

The authors would like to thank Erminia Calabrese, Antony Lewis, James Rich, Adam Riess, and Ashley Ross for helpful discussions and clarifications regarding data sets and software used in this work, and we are also grateful to Adam Riess for reading a draft of the manuscript and providing useful suggestions. We acknowledge the use of the GetDist plotting package ${ }^{19}$

\section{REFERENCES}

19 http://getdist.readthedocs.io/en/latest/
Abazajian, K. N., Arnold, K., Austermann, J., et al. 2015, Astroparticle Physics, 63, 66

Abazajian, K. N., Adshead, P., Ahmed, Z., et al. 2016, ArXiv e-prints, arXiv:1610.02743

Addison, G. E., Hinshaw, G., \& Halpern, M. 2013, MNRAS, 436, 1674

Addison, G. E., Huang, Y., Watts, D. J., et al. 2016, ApJ, 818, 132

Alam, S., Ata, M., Bailey, S., et al. 2017, MNRAS, 470, 2617

Alsing, J., Heavens, A., \& Jaffe, A. H. 2017, MNRAS, 466, 3272

Anderson, L., Aubourg, É., Bailey, S., et al. 2014, MNRAS, 441, 24

Ata, M., Baumgarten, F., Bautista, J., et al. 2017, ArXiv e-prints, arXiv:1705.06373

Aubourg, E., Bailey, S., Bautista, J. E., et al. 2015, Phys. Rev. D, 92,123516

Aver, E., Olive, K. A., \& Skillman, E. D. 2015, J. Cosmology Astropart. Phys., 7, 011

Aylor, K., Hou, Z., Knox, L., et al. 2017, ArXiv e-prints, arXiv:1706.10286

Bautista, J. E., Busca, N. G., Guy, J., et al. 2017, A\&A, 603, A12

Bennett, C. L., Larson, D., Weiland, J. L., \& Hinshaw, G. 2014 ApJ, 794, 135

Bennett, C. L., Larson, D., Weiland, J. L., et al. 2013, ApJS, 208, 20

Bernal, J. L., Verde, L., \& Riess, A. G. 2016, J. Cosmology Astropart. Phys., 10, 019

Betoule, M., Kessler, R., Guy, J., et al. 2014, A\&A, 568, A22

Beutler, F., Blake, C., Koda, J., et al. 2016, MNRAS, 455, 3230

Beutler, F., Blake, C., Colless, M., et al. 2011, MNRAS, 416, 3017

Blomqvist, M., Kirkby, D., Bautista, J. E., et al. 2015, J. Cosmology Astropart. Phys., 11, 034

Bonvin, V., Courbin, F., Suyu, S. H., et al. 2017, MNRAS, 465, 4914

Brust, C., Cui, Y., \& Sigurdson, K. 2017, J. Cosmology Astropart. Phys., 8, 020

Busca, N. G., Delubac, T., Rich, J., et al. 2013, A\&A, 552, A96

Calabrese, E., Hlozek, R. A., Battaglia, N., et al. 2013,

Phys. Rev. D, 87, 103012

Cardona, W., Kunz, M., \& Pettorino, V. 2017, J. Cosmology Astropart. Phys., 3, 056

Carlstrom, J. E., Ade, P. A. R., Aird, K. A., et al. 2011, PASP, 123,568

Cole, S., Percival, W. J., Peacock, J. A., et al. 2005, MNRAS, 362,505

Cooke, R. J., Pettini, M., Jorgenson, R. A., Murphy, M. T., \& Steidel, C. C. 2014, ApJ, 781, 31

Cooke, R. J., Pettini, M., Nollett, K. M., \& Jorgenson, R. 2016 , ApJ, 830, 148

Cuesta, A. J., Vargas-Magaña, M., Beutler, F., et al. 2016, MNRAS, 457, 1770

Cyburt, R. H., Fields, B. D., Olive, K. A., \& Yeh, T.-H. 2016, Reviews of Modern Physics, 88, 015004

Delubac, T., Bautista, J. E., Busca, N. G., et al. 2015, A\&A, 574, A59

Dhawan, S., Jha, S. W., \& Leibundgut, B. 2017, ArXiv e-prints, arXiv:1707.00715

du Mas des Bourboux, H., Le Goff, J.-M., Blomqvist, M., et al. 2017, ArXiv e-prints, arXiv:1708.02225

Dunkley, J., Calabrese, E., Sievers, J., et al. 2013, J. Cosmology Astropart. Phys., 7, 025

Efstathiou, G. 2014, MNRAS, 440, 1138

Eisenstein, D. J., \& Hu, W. 1998, ApJ, 496, 605

Eisenstein, D. J., Zehavi, I., Hogg, D. W., et al. 2005, ApJ, 633, 560

Feeney, S. M., Mortlock, D. J., \& Dalmasso, N. 2017, ArXiv e-prints, arXiv:1707.00007

Fixsen, D. J. 2009, Àp $\bar{J}, 707,916$

Fixsen, D. J., Cheng, E. S., Gales, J. M., et al. 1996, ApJ, 473, 576

Follin, B., \& Knox, L. 2017, ArXiv e-prints, arXiv:1707.01175

Font-Ribera, A., Kirkby, D., Busca, N., et al. 2014, J. Cosmology Astropart. Phys., 5, 027

Freedman, W. L., Madore, B. F., Scowcroft, V., et al. 2012, ApJ, 758,24

Gelman, A., \& Rubin, D. B. 1992, Statist. Sci., 7, 457

Heavens, A., Fantave, Y., Sellentin, E., et al. 2017, ArXiv e-prints, arXiv:1704.03467

Hildebrandt, H., Viola, M., Heymans, C., et al. 2017, MNRAS, 465,1454

Hinshaw, G., Larson, D., Komatsu, E., et al. 2013, ApJS, 208, 19

Hong, T., Han, J. L., \& Wen, Z. L. 2016, ApJ, 826, 154

Hou, Z., Reichardt, C. L., Story, K. T., et al. 2014, ApJ, 782, 74

Izotov, Y. I., Thuan, T. X., \& Guseva, N. G. 2014, MNRAS, 445, 778

Joudaki, S., Blake, C., Heymans, C., et al. 2017, MNRAS, 465, 2033 
Kazin, E. A., Koda, J., Blake, C., et al. 2014, MNRAS, 441, 3524 Köhlinger, F., Viola, M., Joachimi, B., et al. 2017, MNRAS, 471, 4412

Lewis, A., \& Bridle, S. 2002, Phys. Rev. D, 66, 103511

Louis, T., Grace, E., Hasselfield, M., et al. 2017, J. Cosmology Astropart. Phys., 6, 031

Marcucci, L. E., Mangano, G., Kievsky, A., \& Viviani, M. 2016 Physical Review Letters, 116, 102501

Peimbert, A., Peimbert, M., \& Luridiana, V. 2016, Rev. Mexicana Astron. Astrofis. 52, 419

Percival, W. J., Ross, A. J., Sánchez, A. G., et al. 2014, MNRAS, 439, 2531

Pettini, M., \& Cooke, R. 2012, MNRAS, 425, 2477

Planck Collaboration, Ade, P. A. R., Aghanim, N., et al. 2016, A\&A, 594, A13

Planck Collaboration Int. LI. 2016, ArXiv e-prints, arXiv:1608.02487

Planck Collaboration Int. XLVI. 2016, A\&A, 596, A107

Planck Collaboration XI. 2016, A\&A, 594, A11

Reid, B., Ho, S., Padmanabhan, N., et al. 2016, MNRAS, 455, 1553

Riemer-Sørensen, S., Kotuš, S., Webb, J. K., et al. 2017, MNRAS, 468,3239

Riemer-Sørensen, S., \& Sem Jenssen, E. 2017, ArXiv e-prints, arXiv:1705.03653
Riess, A. G., Macri, L., Casertano, S., et al. 2009, ApJ, 699, 539 -. 2011, ApJ, 730, 119

Riess, A. G., Macri, L. M., Hoffmann, S. L., et al. 2016, ApJ, 826, 56

Ross, A. J., Samushia, L., Howlett, C., et al. 2015, MNRAS, 449, 835

Sherwin, B. D., van Engelen, A., Sehgal, N., et al. 2016, ArXiv e-prints, arXiv:1611.09753

Sievers, J. L., Hlozek, R. A., Nolta, M. R., et al. 2013, J. Cosmology Astropart. Phys., 10, 60

Slosar, A., Iršič, V., Kirkby, D., et al. 2013, J. Cosmology Astropart. Phys., 4, 026

Story, K. T., Reichardt, C. L., Hou, Z., et al. 2013, ApJ, 779, 86

Thornton, R. J., Ade, P. A. R., Aiola, S., et al. 2016, ApJS, 227, 21

van Engelen, A., Keisler, R., Zahn, O., et al. 2012, ApJ, 756, 142

Veropalumbo, A., Marulli, F., Moscardini, L., Moresco, M., \& Cimatti, A. 2016, MNRAS, 458, 1909

Wang, Y., Xu, L., \& Zhao, G.-B. 2017, ArXiv e-prints, arXiv:1706.09149

Weinberg, D. H., Mortonson, M. J., Eisenstein, D. J., et al. 2013, Phys. Rep., 530, 87

Zhang, B. R., Childress, M. J., Davis, T. M., et al. 2017, MNRAS, 471, 2254 